\title{
Why did the COVID-19 Epidemic Stop in China and does not Stop in the Rest of the World? (Application of the Two-Component Model)
}

\author{
Juri Dimaschko ${ }^{1 *}$, Vladimir Shlyakhover ${ }^{2}$, Mykola Iabluchanskyi ${ }^{3}$ \\ ${ }^{I}$ Technische Hochschule Lübeck, Lübeck, Germany. \\ ${ }^{2}$ Department of Cardiology, Barzilai Medical Center, Ashkelon Faculty of Health Sciences, Ben-Gurion University of the Negev, Beer- \\ Sheva, Israel. \\ ${ }^{3}$ Department of Internal Medicine, V. N. Karazin Kharkiv National University, Kharkiv, Ukraine.
}

Received 09 February 2021; Revised 19 March 2021; Accepted 03 April 2021; Published 01 June 2021

\begin{abstract}
The vastly different courses of the COVID-19 epidemic in China and the rest of the world are investigated and explained within two-component epidemic model. The model is based on separate accounting for the contribution to the epidemic from two types of immune response to a viral infection - innate and adaptive immunity. Any infected person becomes asymptomatic with probability $(1-p)$ or symptomatic with probability $p$. In the first case, innate immunity is sufficient to protect a person. In the second case, innate immunity is insufficient, and adaptive immunity comes into play. In the asymptomatic state, the person remains outwardly healthy, mobile and can spread the infection. In the symptomatic state, the person becomes ill, isolated and cannot spread the infection. We assume that the contribution to the epidemic process from asymptomatic carriers is dominant in comparison with the contribution from the usual incubation period in the symptomatic state. The key parameters of the model are the virus lifetime $T$ in the asymptomatic state and the spread rate $\beta$. At moderate $\beta T$ values, the model describes a long, slowly decreasing morbidity plateau, which transforms into wavelike solution at $\beta T \gg 1$. In the case of $\beta T \rightarrow \infty$, which corresponds to a stable non-pathogenic strain, the model solution is limited to single wave only. We believe that the spread of such a non-pathogenic strain and its subsequent dominance is responsible for ending the epidemic after the single wave of incidence in China. A way to stop the epidemic in the rest of the world may consist in displacing the circulating pathogenic virus with its stable non-pathogenic strain.
\end{abstract}

Keywords: COVID-19; Epidemic Model; Waves of Incidence; Immune Response; Asymptomatic Infection; Latency.

\section{Introduction}

The COVID-19 epidemic has divided the world into two unequal parts - China and the rest of the world. In China, the epidemic developed in the usual way: an exponential increase in the incidence, then a pronounced maximum, then a decline and the end of the epidemic. The whole cycle took about two and a half months. In the rest of the world, the epidemic was completely different. After a stage of exponential growth in daily morbidity, an inexplicable plateau has entered in many countries. Then the second and third waves emerged, the epidemic is currently ongoing, and it is unclear how many more waves may follow. Nothing of the kind is observed in China.

\footnotetext{
* Corresponding author: dimaschko@gmx.net

do) http://dx.doi.org/10.28991/SciMedJ-2021-0302-2

$>$ This is an open access article under the CC-BY license (https://creativecommons.org/licenses/by/4.0/).

(C) Authors retain all copyrights.
} 
In previous Dimaschko (2020) [1, 2] works, it was shown that the asymmetric course of the epidemic with a long plateau after a pronounced maximum can be explained by the presence of asymptomatic carriers of infection - superspreaders. However, this model did not explain the emergence of repeated waves of the epidemic. In addition, the nature of the asymptomatic state remained unclear. Finally, the question of the difference in the courses of the epidemic in China and in the rest of the world was not raised in any way.

In this paper, the division of the infected into asymptomatic and symptomatic is substantiated. We associate this with two types of immunity - innate and adaptive. If the innate immunity is sufficient to limit and subsequently eliminate the virus, then the person becomes the asymptomatic carrier of the virus. If the innate immunity is not enough for this, then the person becomes ill, and adaptive immunity comes into play. The choice of the type of reaction is determined by the probability $p$ with which an infected person will get sick. This probability gives an idea of the degree of pathogenicity of the virus.

With a probability $(1-p)$ innate immunity is sufficient, and the infected person remains practically healthy. Then the adaptive immunity is not activated. In this case, the infection remains in the body for a long time, and all this time the infected person can spread the infection. This time can be incorporated in the model either directly by the lifetime $T$ of the virus in the body of an asymptomatically infected person (the concept of the lifetime), or by the elimination rate $\omega \sim 1 / T$ (the concept of the elimination rate).

The article is structured as follows. In the next part, we look at the symptomatic and asymptomatic states as two modes of immune response to the virus. Response factors relevant to the two-component model are discussed here. In the third part, on the basis of these factors, the two-component model and its dynamic equations are built, the dependence of the course of the epidemic on the parameters of the model is investigated. Comparing the solution with the observed course of the pandemic allows us to determine the values of three parameters of the model. In the fourth part, the factor of the lifetime of the virus, which leads to a sequence of incidence waves, is considered separately. This makes it possible to interpret the incidence waves observed during the pandemic, as well as to explain the significant difference between the Chinese and world cases. The fifth part assesses the possible impact of virus mutations and parameter drift on the course of the epidemic. In the final part, we discuss the results obtained and consider an alternative approach to ending the epidemic, not associated with mass vaccination.

\section{Symptomatic and Asymptomatic States}

The biological characteristics of viruses and their interaction with multicellular organisms, despite numerous studies, remain poorly understood. The complexity of the problem is determined by the huge number of viruses, their constant variability, and the transition from one species of organisms to another, as happened with the virus that causes COVID-19. To create a mathematical model for the development of a pandemic, we used the following provisions on the interaction of the virus and the human body, which, in our opinion, do not contradict existing ideas.

- After entering the body, the virus begins to multiply;

- Innate/background immunity triggers nonspecific defence agents;

- Under certain conditions, this turns out to be sufficient and equilibrium occurs at a safe level, which does not lead to the development of symptoms of infection, i.e., diseases. We call further this hidden infectious period as latency period (Figure 1). Since this is an equilibrium state, the system organism + virus can stay in it for a long time without any external manifestations. It is this state that we call asymptomatic. In the asymptomatic state, the person is infected but not sick;

- If the background immunity turns out to be insufficient to establish a balance at a safe level, then the body begins to prepare an adaptive immune response in advance. After passing a certain threshold level of infection, also shown in Figure 1, symptoms develop, and the person becomes ill. In this state, the main role is no longer played by the background immunity, but by the specific immunity. It is it which is responsible for limiting the number of viruses and recovery. It is this state that we call symptomatic;

- The type of immune response is determined at some time point in the development of the infection, which we will call the bifurcation point. After it, either a symptomatic state develops (with a certain probability $p$ ), or an asymptomatic state (with a probability $1-p$ ).

For some time preceding the disease, when the background immunity has already exhausted its means and the virus continues to multiply, but the symptoms have not yet appeared, the person is also an asymptomatic carrier. This state is non-equilibrium and therefore relatively short-lived. This is the incubation period (see Figure 1).

Thus, it is natural to expect that the incubation period is relatively short, and the latency period is long. Since during the period of illness the persons are isolated and cannot spread the infection, they can do this either in the incubation or in the latency phase. Which of these two phases is critical for the spread of the virus? 


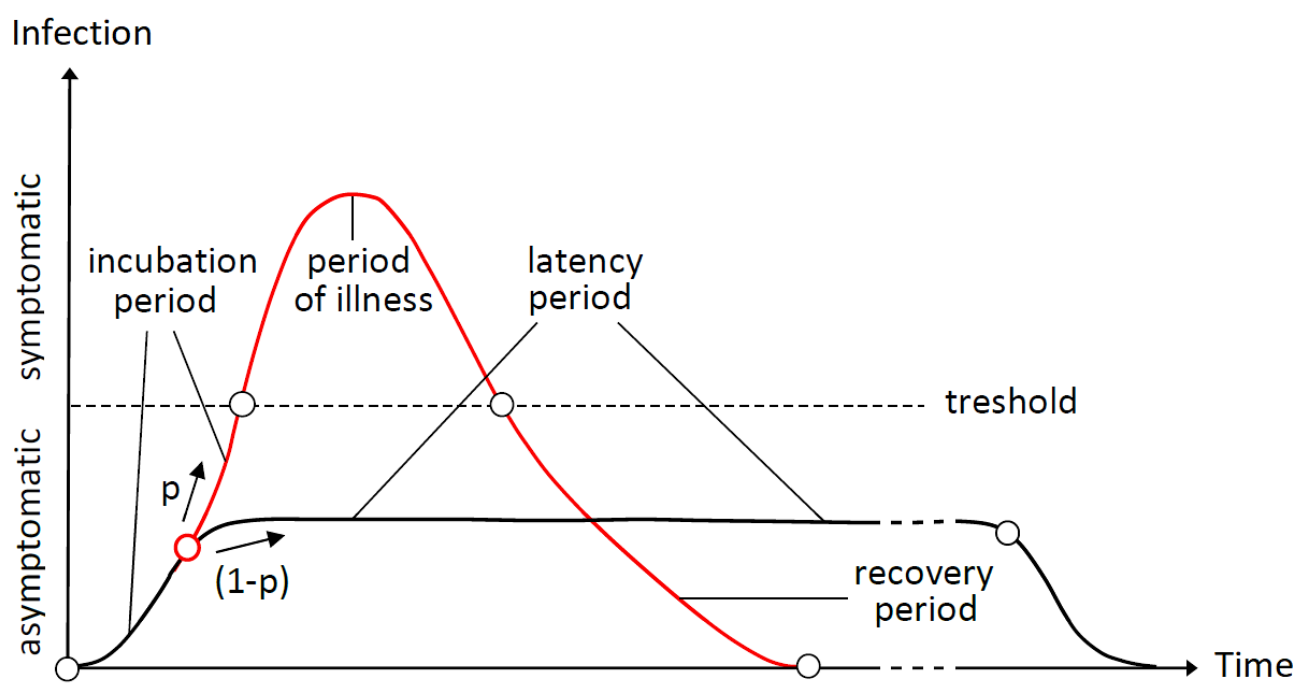

Figure 1. Symptomatic and asymptomatic pathways of interaction with infection. The bifurcation point is shown with a red circle. After that, there is transition either to the symptomatic state with probability $p$ or to the asymptomatic state with probability $(1-p)$. In the first case (red line), the infected persons go through the incubation period, period of illness and recovery period. In the second case (black line), they only go through the latency period.

On the one hand, the latency period is much longer, which increases the contribution of this phase to the spread. On the other hand, in the incubation phase, the number of viruses in the body is much higher, which somehow evens the chances. Therefore, the key to answering the question posed is the balance of probabilities at the bifurcation point, also shown in Figure 1: what is the ratio of the probabilities of transition to the short-term incubation and to the longterm latent phase $(p$ and $(1-p)$, respectively)?

In our work, we assume that the transition to the latency phase is more likely. Comparison of the results obtained in the model with the course of the COVID-19 pandemic fully confirms this assumption a posteriori. The transition to the latency phase turns out to be three or more orders of magnitude more probable than the incubation phase, i.e. $p \sim 10^{-3}$.

Thus, in our model, we will neglect the contribution from the incubation period to the spread of infection. Under these conditions, there are only two parameters that describe the development of infection in a person infected with the virus and are important for the further transmission of the virus. These are the probability $p$ of the transition to a symptomatic state and the duration $T$ of the latency period. Along with conventional spread rate $\beta$, they form three basic parameters of the model under consideration

\section{Two-component Model: Random Decay Approach}

The two-component epidemic model is based on following assumptions:

- Depending on the effectiveness of the nonspecific immune response, the level of infection exceeds the safety threshold and becomes dangerous (with probability $p$ ) or does not exceed it and remains safe (with probability $1-p$ ). In the first case, adaptive/specific immunity turns in play and symptoms of the disease appear. In the second case, this does not happen, the infected person remains healthy, but the infection for a long time $T$ remains in the body in the latent form. In the first case, there is a symptomatic, in the second asymptomatic course of the infection;

- When symptoms appear, the infected persons become isolated and cannot_spread the infection. If they recover, they acquire ideal long-term immunity and cannot be infected anymore;

- The asymptomatic infected persons do not become isolated and continues to spread the infection at some rate $\beta$. They remain active during the entire latency period $T$, until the virus is cleared from the body. The corresponding elimination rate is $\omega \sim 1 / T$. After removing the virus from the body, the asymptomatic infected persons return to the uninfected state. They do not acquire long-term immunity and can be reinfected;

- Due to the appearance of long-term immunity, the transition to the symptomatic state is irreversible. On the contrary, due to the absence of the appearance of long-term immunity, the transition to the asymptomatic state is reversible. This means that after the hidden infectious period, the infected person returns to his original susceptible state.

These considerations lead to the epidemic pattern shown in Figure 2. 


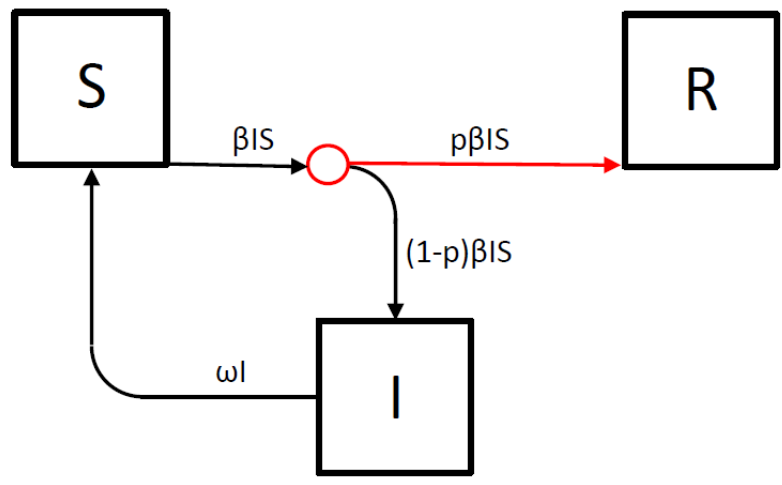

Figure 2. Two-component epidemic diagram. Here $S$ is the share of susceptible, $I$ is the share of asymptomatically infected, $R$ is the share of symptomatically infected + the share of those who recovered after the illness and got the long-term immunity. Model parameters: $\omega$ is the elimination rate, $\beta$ is the spread rate, $p$ is the relative probability of symptomatic infection, $(1-p)$ is the relative probability of asymptomatic infection. The bifurcation point is shown with red circle.

It is like the conventional SIR model and differs from it in the meaning of the variables. A distinctive feature of the two-component model is the bifurcation point, at which it is possible to choose between the symptomatic state (disease) and the asymptomatic state (latent infection). Another feature is the presence of a closed SIS cycle of reinfection with the participation of asymptomatic infected. The reason for the cycle is the absence of long-term immunity after asymptomatic infection. Neither of these two features exist in the conventional SIR model, which ignores the possibility of asymptomatic infection.

The dynamic equations of the two-component model directly follow from its epidemic scheme and have the form:

$\frac{d S}{d t}=-\beta I S+\omega I$

$\frac{d I}{d t}=(1-p) \beta I S-\omega I$

$\frac{d R}{d t}=p \beta I S$

Note that in these equations, the elimination of viruses is a random decay process with a decay rate of $\omega$. We call this random decay approach. An alternative approach using the virus lifetime T, when Equation 2 looks different, will be considered in the next section.

In view of $d R / d t>0$ the asymptotic behaviour of any its solution as $t \rightarrow \infty$ is $(S, I, R) \rightarrow(0,0,1)$, that is, for a sufficiently long time, the entire population must go through the disease and acquire long-term immunity. Like the SIR model [3], the two-component model has an exact parametric solution. In what follows, however, we will only be interested in the limit of the low probability of a symptomatic course, when $p \ll 1$. Then, to calculate the daily incidence $d R / d t$ it is sufficient to use the exact analytical solution in the case $p=0$ :

$I(t)=\frac{I_{0}\left(1-\frac{\omega}{\beta}\right)}{I_{0}+\left(1-\frac{\omega}{\beta}-I_{0}\right) e^{(\omega-\beta) t}}$

$S(t)=1-I(t)$

satisfying the initial condition $I(0)=I_{0}, S(0)=1-I_{0}$. When $t \rightarrow \infty$ the share of asymptomatic carriers $I(t)$ tends to the limit:

$\lim _{t \rightarrow \infty} I(t)=1-\frac{\omega}{\beta}$

In the limiting case $p \ll 1$, the daily incidence $d R / d t$ is expressed through $I(t)$ and $S(t)$ by the relation:

$\frac{d R}{d t}=p \beta I(t) S(t)$

In this limiting case, the asymptotic of daily incidence has the form: 
$\lim _{t \rightarrow \infty}\left(\frac{d R}{d t}\right)=p \omega\left(1-\frac{\omega}{\beta}\right)$

The incidence as a function of $\omega$ reaches maximum at $\omega=\beta / 2$, the corresponding maximal incidence is:

$\left(\frac{d R}{d t}\right)_{\max }=\frac{1}{4} p \beta$

The time course of daily incidence depends on the ratio $\omega / \beta$. When $\omega / \beta>1 / 2$, the incidence monotonously increases to the asymptotic value (8). At $\omega / \beta \leq 1 / 2$, the incidence first passes through the maximum (9), and then decreases to the asymptotic value (8). It is this case, which is of most interest for what follows, is shown in Figure 3.

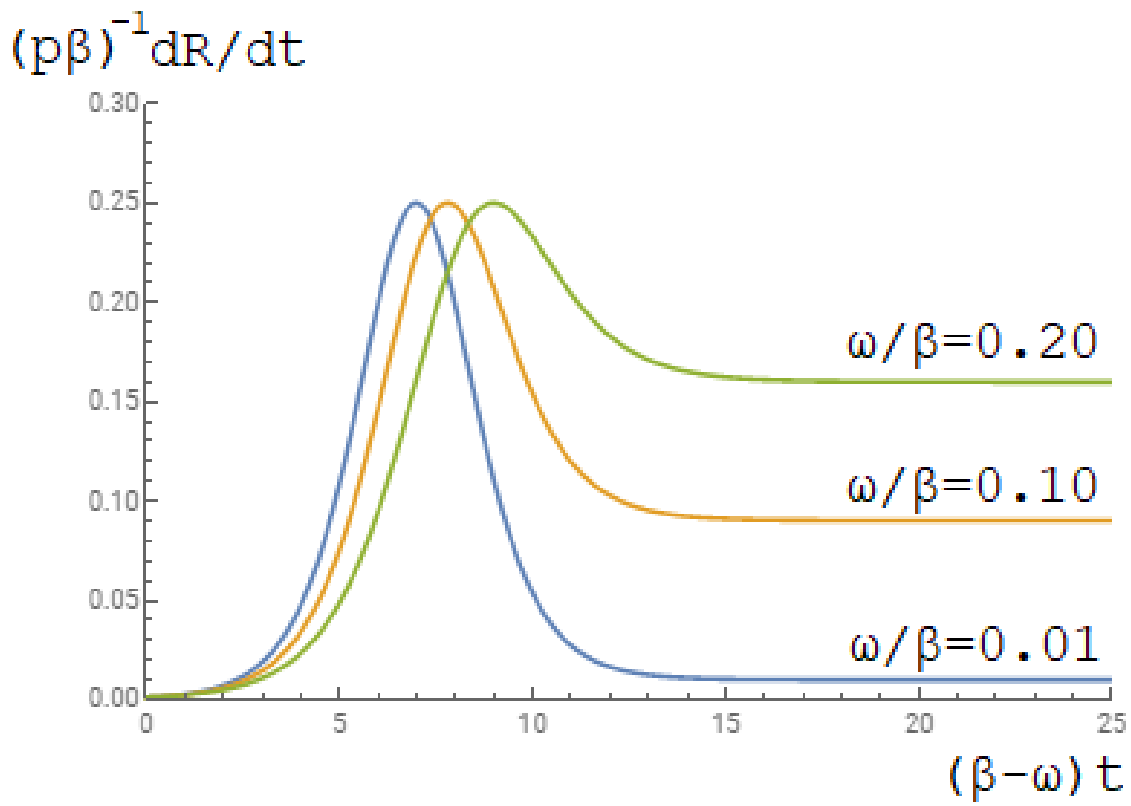

Figure 3. The course of daily incidence $d R / d t$ in the random decay approach, depending on the ratio $\omega / \beta$

Obviously, since the value of $R$ is bounded above by unit, over time it can no longer increase. Hence for $t \rightarrow \infty$ the approximation of $p \ll 1$ does not work anymore, and the flat plateau (8) gradually goes down to zero:

$\lim _{t \rightarrow \infty}\left(\frac{d R}{d t}\right)=0$

as it should be. According to (9), the characteristic decay time of the epidemic cannot be less than:

$T_{t o t}=\frac{4}{p \beta}$

Now we can compare the results obtained with the observed course of the COVID-19 pandemic. Since the case criteria change over time, the most accurate picture is given by daily mortality values. At the same time, the true incidence is obtained from mortality by dividing it by the fatality rate which is currently $2.2 \%$ [4].

As the course of the epidemic in China and in the rest of the world differs significantly, we fit data of China and the rest of the world separately. Fitting in both cases is shown in Figures 4 and 5. The results are following values of parameters in China:

$\beta=0.16 d^{-1}, \quad \omega=1.4 \cdot 10^{-3} d^{-1}, \quad p=3 \cdot 10^{-4}$,

and in the rest of the world:

$\beta=0.20 d^{-1}, \omega=4 \cdot 10^{-2} d^{-1}, \quad p=10^{-3}$.

Thus, the pandemic data directly confirm the above assumption about the smallness of the pathogenicity parameter $p$, the value of which indeed does not exceed $10^{-3}$. 


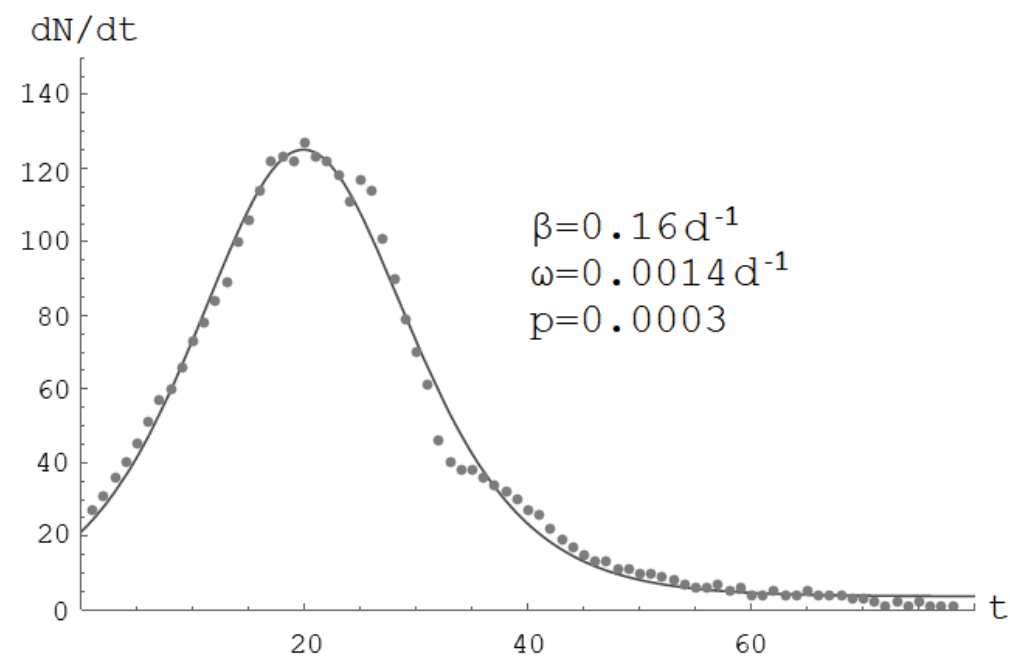

Figure 4. Daily deaths in the COVID-19 epidemic in China versus time (dots) and model dependence (solid line)

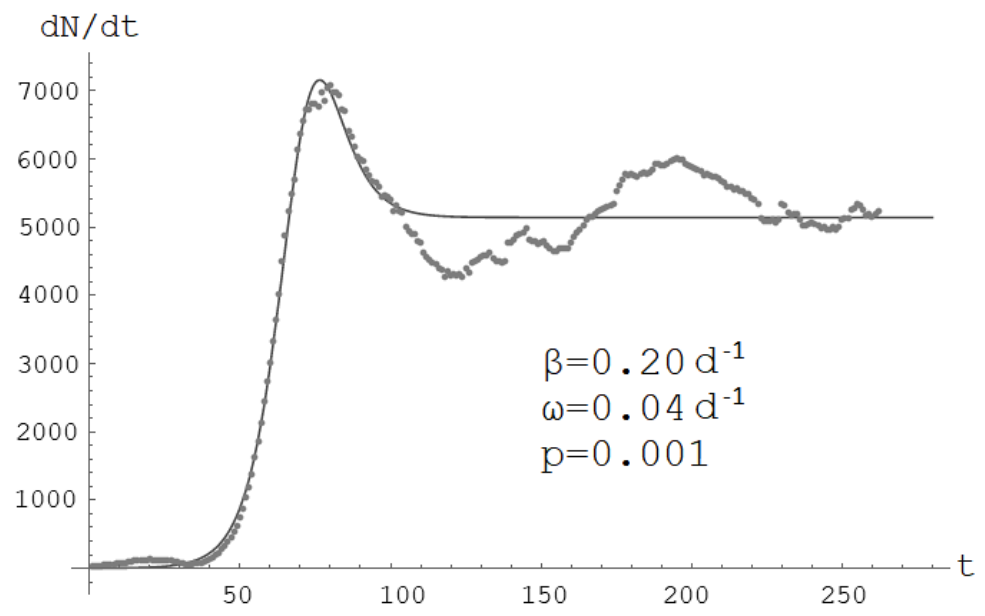

Figure 5. Daily deaths in the COVID-19 world pandemic versus time (dots) and model dependence (solid line)

In the China case attention is drawn to the anomalously small value of the elimination rate $\omega$. Whereas, according to world data, the virus remains in the body of asymptomatic infected $1 /\left(4 \cdot 10^{-2}\right)=25$ days, according to the Chinese epidemic data this time is already $1 /\left(1.4 \cdot 10^{-3}\right)=714$ days, or 2 years.

Together with the markedly lower pathogenicity parameter $p$ in China, this suggests that a different virus strain became dominant as the epidemic spread across China compared to the rest of the world. According to the parameters found, this strain should be non-pathogenic and long-lived in the human body.

Perets \& Perets (2020) [8] already considered the hypothesis of the existence of a low pathogenic virus in the prehistory of the COVID-19 epidemic as the cause of an abnormally low incidence in China. However, there it was assumed only as a temporary factor that led to the activation of adaptive immunity in the population. On the other hand, the existence of non-pathogenic strains of coronavirus is a well-known fact [9, 10], and it can be expected among the mutations of the original SARS-CoV-2 virus.

Next, in accordance with Equation 6 the asymptomatic carriers are absolute majority of the population. In the world beyond China their equilibrium share $I(\infty)=(1-\omega / \beta)$ is about $80 \%$, and in China it is close to $100 \%$. It should be noted that this value does not have to be reflected in the test results if the PCR testing is tuned to a higher level of virus presence corresponding to the onset of the disease. Apparently, the largest contribution to the spread of the virus is made by healthy, younger infected with stronger innate immunity and more mobility.

Using the found values of the spread rate and the pathogenicity parameter, we estimate the expected duration of a pandemic by Equation 11:

$T_{\text {tot }}=2 \cdot 10^{4} d$,

that is about 50 years. Naturally, this estimation ignores possible mutations of the virus and vaccination. 


\section{Virus Lifetime and Waves of Incidence}

The scheme considered in the previous section treats virus elimination as a statistical process with a decay rate $\omega$. A more detailed description assumes considering the individual lifetime of the virus, determined by some function $g(t)$. It is the probability that the virus remains viable after $t$ days in the body of a healthy infected person. In this context, the term "healthy" means the action of only nonspecific innate immunity and the absence of the need for an adaptive immune response. 6:

The function $g(t)$ must meet the obvious conditions $g(0)=1, g(\infty)=0$. Its possible shapes are shown in Figure

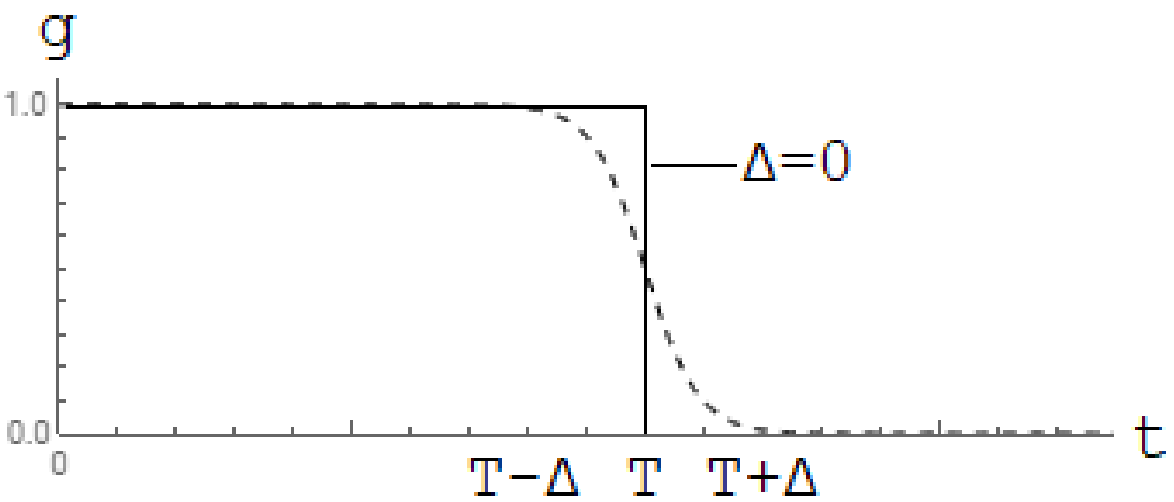

Figure 6. Virus lifetime function. Dashed line approximates the lifetime function by Fermi dependence $g(t)=(1+$ $\left.e^{(t-T) / \Delta}\right)^{-1}$ and solid line corresponds to the step-like limiting case $\Delta=0$ which is the Heaviside theta function $g(t)=$ $\boldsymbol{\theta}(\boldsymbol{T}-\boldsymbol{t})$.

In this approach, the differential Equation 2 of the system (1-3) is replaced by integral one

$I(t)=(1-p) \beta \int_{0}^{t} g(t-\tau) I(\tau) S(\tau) d \tau$

and becomes non-local. In the limiting case $p \ll 1$ of interest to us, the system of equations is, however, significantly simplified. In this limit, the incidence of $d R / d t$ is related to the rate of infection $d I / d t$ :

$\frac{d R}{d t}=p \frac{d I}{d t}$

The $I(t)$ function now must be found from the single equation:

$I(t)=\beta \int_{0}^{t} g(t-\tau) I(\tau)[1-I(\tau)] d \tau$

If we restrict ourselves to the simplest case of the step dependence $g(t)=\theta(T-t)$ (see Figure 6), then this equation is reduced to a differential one with retarded argument:

$\left.\frac{d I}{d t}\right|_{t}=\beta\left\{\left.[I(1-I)]\right|_{t}-\left.[I(1-I)]\right|_{t-T}\right\}$

This equation is easy to solve numerically. A remarkable feature of its solution is the appearance of incidence waves as shown in Figure 7. These waves appear at large enough values of the dimensionless product $\beta T$.

The meaning of the waves is obvious. The incidence is a consequence of cyclical re-infection. After the first wave of infection has passed, the number of susceptible people decreases, and hence the incidence. Over time, T, firstgeneration viruses begin to be eliminated and the proportion of susceptible ones increases. As a result, the epidemic process begins again, a second wave of re-infection occurs, new infected appear, infected with second-generation viruses, etc. 

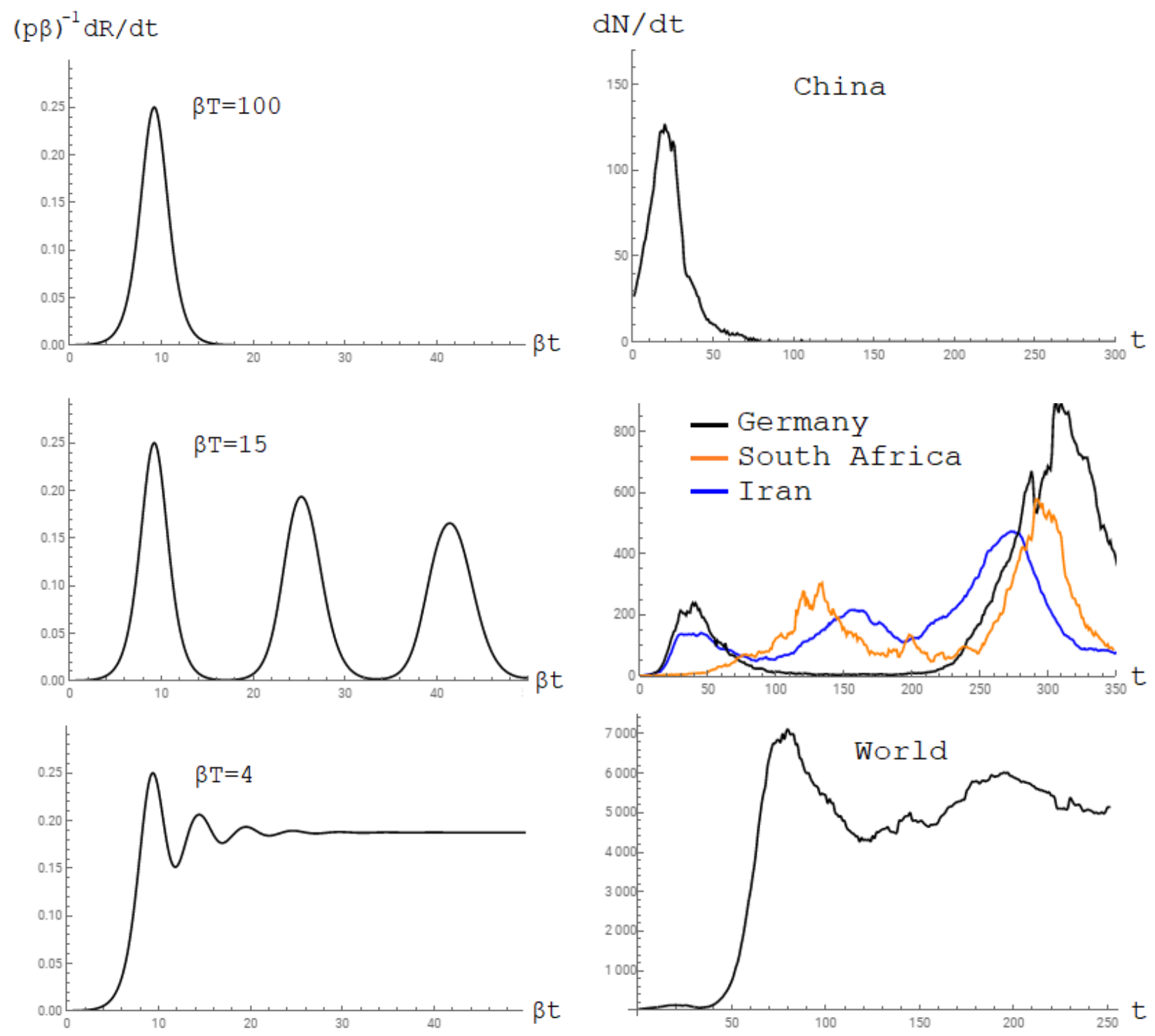

Figure 7. Waves of daily incidence $d R / d t=p d I / d t$ depending on the value of $\beta T$. The wave solutions are qualitatively compared with death rate curves from China, from three selected countries (Germany, South Africa, Iran), and from the total world [4].

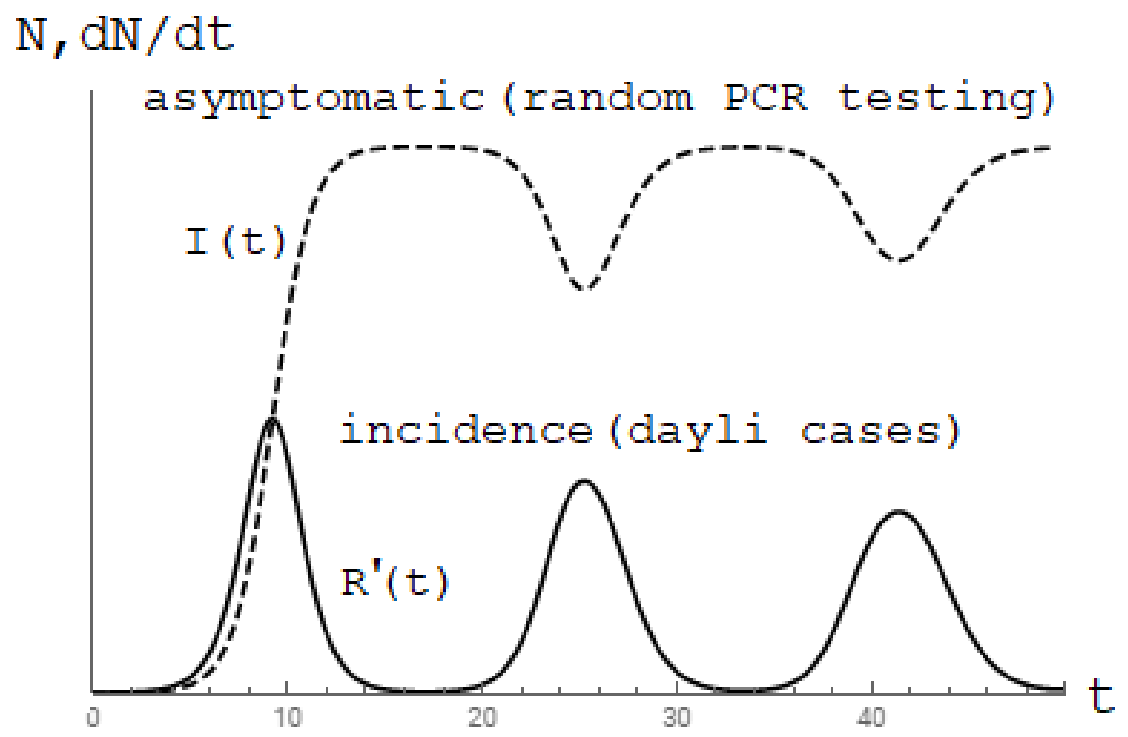

Figure 8. Share of asymptomatic infected $I(t)$ and incidence $R^{\prime}(t)$ are oscillating in antiphase 
Simultaneous evolution of both share of asymptomatic infected $I(t)$ and incidence $R^{\prime}(t)$ in the wave-like solution is shown in Figure 8. As the viruses become eliminated, the number of asymptomatic infected decreases, instead new susceptible arise and therefore the incidence increases. In such a way the share of asymptomatic and incidence are oscillating in antiphase. These fluctuations are possible because the change in the proportion of asymptomatic infections $I$ is reversible.

The proportion of asymptomatically infected people is difficult to measure, since the level of infection may be below the sensitivity threshold of PCR tests, and the localization of viruses may be outside the nasopharyngeal mucosa. Nevertheless, this result can explain an inversion that is often observed during the COVID-19 epidemic, when, despite the decrease in the proportion of positive PCR tests, an increase in the incidence occurs. This happens when daily testing far exceeds the daily incidence. During this period, testing is mostly random, and the proportion of positive tests is roughly proportional to the share $I$ of those infected without symptoms

Thus, the two-component model explains the incidence waves by cycles of re-infection of the population, the period of which is determined by the time of virus elimination in asymptomatic infected. A small proportion of those reinfected fall ill, which manifests as a wave of incidence.

The wave-like solution gives a simple geometric meaning to all three parameters of the model, as shown in Figure 9. The virus lifetime $T$ means the distance between the waves, the width of the waves is inversely proportional to the spread rate $\beta$, and the area under plot of one wave is directly proportional to the parameter $p$, which corresponds to degree of pathogenicity of the virus.

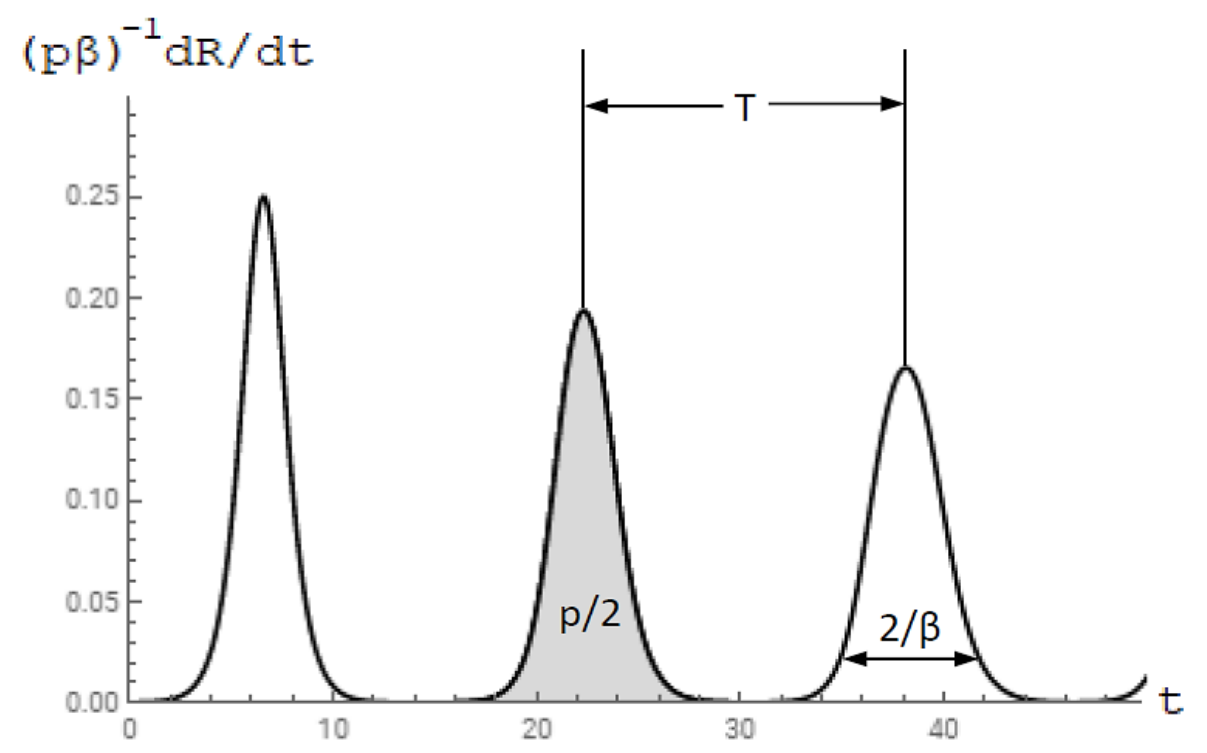

Figure 9. The meaning of the $T, p$ and $\beta$ parameters of the two-component model

The waves are smoothed out if their width $(2 / \beta)$ becomes of the order of the distance between them $(T)$, i.e., at $\beta T<2$. This tendency is visible in Figure 7. The same effect on the incidence waves also causes the lifetime formfactor $\Delta$ shown above in Figure 6.

A similar averaging of waves occurs when adding data from different countries. In this case, the geographical factor acts [11], which can be seen in the example of the total data on the pandemic in the world (see Figure 7). Therefore, it is natural to assume that the averaging of waves in the total data of the pandemic shown in Figure 7 is caused not by the lower value of the $\beta T$ parameter, but by the addition of epidemic waves from many regions of the world.

Anyway, high value of the spread rate $\beta$ is a necessary condition for the emergence of the incidence waves. Otherwise, the waves are smoothed out and transform into a plateau that appears in the random decay approach considered in the previous section (see Figure 3). The relationship between virus lifetime $T$ in the detailed virus lifetime approach and elimination rate $\omega$ in the random decay approach is given by $\omega=1 / T$.

In our opinion, the most important of the obtained family of wave solutions is the simplest single-wave solution corresponding to the $\beta T \rightarrow \infty$ limit. We assume that it describes the epidemic situation in China (as shown in Figure 7 ). The absence of repeated waves indicates a high degree of stability of the dominant strain here $(T \rightarrow \infty)$, and an extremely low morbidity means its non-pathogenicity $(p \rightarrow 0)$. 
The question of the nature of such a non-pathogenic strain requires virological research. If detected, it seems reasonable to provide its spread to displace the less stable and more pathogenic strains that are shaping the current course of the global pandemic.

\section{Modulation and Drift of Parameters}

So far, we have assumed all three parameters of the model to be constant. However, over time, epidemic factors change, which leads to a change in the parameters. If the rate of this change is much lower than the spread rate $\beta$, then the same solutions can be used, simply by replacing constant parameters with the corresponding functions of time, considering the drift of epidemic factors.

The values of the parameters $(12,13)$ found above should be interpreted as averages over a certain period. However, these values may change in the future.

Epidemic factors are influenced by both measures taken to reduce the incidence [2] and changes in the properties of the virus itself due to mutations [12]. Virus mutations can affect all three parameters $\beta, \omega$ and $p$. In addition, the spread rate $\beta$ can be temporarily reduced due to anti-epidemic measures taken, for example, during lockdown [2].

The pathogenicity $p$ of the virus depends on the average level of innate nonspecific immunity in the population. Typically, this level changes cyclically throughout the year, decreasing in winter and rising in summer. This seasonal modulation of the immunity leads to flu epidemics in the fall and winter.

Since according to the Equation 9 the maximum value of the incidence is $p \beta / 4$, the sanitary measures including lockdown and thereby reducing the spread rate of $\beta$ indeed reduce the incidence. However, the currently observed winter wave is much higher than the previous winter wave. This may be a consequence of an increase in the pathogenicity parameter $p$ due to decrease in the average level of nonspecific immunity. If this is indeed the case, and the reason for this effect is lifestyle changes due to isolation, then we must take this into account and limit the use of these types of measures. Because too long lockdowns could cause long-time negative drift in the average immunity level.

\section{Discussion}

The two-component model constructed in our work describes the interaction with infection as a fundamentally random process. The further nature of this process is determined soon after infection, at the point of bifurcation. After passing this point, the infected person either gets sick or becomes an asymptomatic carrier.

It is worth to note that virus infection plays a twofold role in the asymptomatic state. On the one hand, it can be transmitted further, which contributes to the development of the epidemic. On the other hand, it protects the asymptomatic infected from an immediate symptomatic infection, that is, from disease, since the innate immunity is already in a mode that keeps the infection at a safe level.

In such way, for the latent period $T$ nonspecific innate immunity guarantees the same effective protection against disease as antibodies. However, after elimination of the virus and re-infection, the process may go differently, and the person may get sick. Unlike protection by antibodies, the duration of which is determined by the time of their preservation, in this case the duration of protection is determined by the lifetime $T$ of the virus itself.

In Shaman \& Galanti (2020) and Linde et al. (2009) [13, 14] studies, the idea of temporary protection from virus by another virus was considered in the case of two alternating epidemics with two different viruses. In our model, there are not two different viruses, but we take into account two types of immune response to the same coronavirus.

Of course, the probability $p$ of getting sick, as well as two other parameters of the model, depend on the individual state of the immune system of each infected person. In this paper, we consider the simplest approximation, when this dependence is ignored, and the parameters of the model are taken as averages for the entire population.

Apparently unique feature of the COVID-19 epidemic is that an infection level that is safe for absolute majority of the population is unsafe for the population as a whole and leads to an epidemic spread of the disease. The reason for this is, on the one hand, an exceptionally low likelihood of a symptomatic course, and on the other hand, a high degree of infectiousness. The two-component model assumes that the vast majority of those infected are asymptomatic. However, any of them, having come out of this state and re-infected, can get sick.

Unlike a regular flu epidemic, in which the same people carry the virus and get sick, in the COVID-19 epidemic, some people carry the virus and others get sick. 


\section{Conclusions}

Based on the two-component model, we can draw the following conclusions.

- During the COVID-19 epidemic, the vast majority of the population is infected and is in a hidden asymptomatic phase. A small proportion of those infected become ill and go through the symptomatic phase;

- The COVID-19 virus is permanently circulating in the population, which manifests itself either in the form of a plateau of incidence or in the form of waves. After elimination of the virus from the asymptomatic infected persons, they may become ill as a result of subsequent re-infection;

- The two-component model shows the share of asymptomatic infected in the population to be oscillating in antiphase with symptomatic morbidity. This result explains the inversion often observed during the COVID-19 epidemic, when, despite a decrease in the share of positive PCR tests, an increase in morbidity occurs [4];

- Without taking any measures, the natural duration of the epidemic can be decades;

- The almost complete cessation of the COVID-19 epidemic in China indirectly indicates the widespread in China of a stable, non-pathogenic strain that arose at the early stage of the epidemic due to mutation. Its presence could provide effective protection against infection by the original pathogenic virus. If such a strain were found, it would be wise to use the targeted spread of this strain to effectively end the epidemic in other countries of the world, as has already happened in China.

Since now new pathogenic strains appear and vaccination is faced with a number of problems, the proposed method may turn out to be relevant. Therefore, virological research aimed at identifying the non-pathogenic strain is advisable right now.

\section{Declarations}

\subsection{Author Contributions}

Conceptualization, J.D., V.S. and M.I.; software and formal analysis, J.D.; writing-original draft preparation, J.D.; writing-review and editing, J.D. V.S. and M.I. All authors discussed the results and commented on the manuscript. All authors have read and agreed to the published version of the manuscript.

\subsection{Funding}

The authors received no financial support for the research, authorship, and/or publication of this article.

\subsection{Acknowledgements}

We express our gratitude to Prof. Matteo Ferensby for fruitful discussion of the results, and to Dr. Daniel Genin for help in use of Mathematika tools.

\subsection{Ethical Approval}

The manuscript does not contain experiments on animals and humans; hence ethical permission not required.

\subsection{Data Availability Statement}

Data was obtained from "Our World in Data" and are available in a publicly accessible repository [4].

\subsection{Conflict of Interest}

The authors declare that they have no known competing financial interests or personal relationships that could have appeared to influence the work reported in this paper.

\section{References}

[1] Dimaschko, J. (2020). Superspreading as a regular factor of the COVID-19 pandemic: I. A two-component model. doi:10.1101/2020.06.29.20138008

[2] Dimaschko, J. (2020). Superspreading as a regular factor of the COVID-19 pandemic: II. Quarantine measures and the second wave. doi:10.1101/2020.08.14.20174557.

[3] Harko, T., Lobo, F. S. N., \& Mak, M. K. (2014). Exact analytical solutions of the Susceptible-Infected-Recovered (SIR) epidemic model and of the SIR model with equal death and birth rates. Applied Mathematics and Computation, 236, 184-194 doi:10.1016/j.amc.2014.03.030.

[4] Our World in Data. Available online: https://ourworldindata.org/coronavirus-data-explorer (accessed on January 2021). 
[5] Saad-Roy, C. M., Wingreen, N. S., Levin, S. A., \& Grenfell, B. T. (2020). Dynamics in a simple evolutionary-epidemiological model for the evolution of an initial asymptomatic infection stage. Proceedings of the National Academy of Sciences, 117(21), 11541-11550. doi:10.1073/pnas.1920761117.

[6] Robinson, M., \& Stilianakis, N. I. (2013). A model for the emergence of drug resistance in the presence of asymptomatic infections. Mathematical Biosciences, 243(2), 163-177. doi:10.1016/j.mbs.2013.03.003.

[7] Kemper, J. (1978). The effects of asymptomatic attacks on the spread of infectious disease: A deterministic model. Bulletin of Mathematical Biology, 40(6), 707-718. doi:10.1016/s0092-8240(78)80003-2.

[8] Perets, H. B., \& Perets, R. (2020). A preceding low-virulence strain pandemic inducing immunity against COVID-19. arXiv preprint arXiv:2007.07154.

[9] Enjuanes, L., Sola, I., Almazan, F., Ortego, J., Izeta, A., Gonzalez, J. M., ... Sanchez, C. (2001). Coronavirus derived expression systems. Journal of Biotechnology, 88(3), 183-204. doi:10.1016/s0168-1656(01)00281-4.

[10] de Haan, C. A. M., van Genne, L., Stoop, J. N., Volders, H., \& Rottier, P. J. M. (2003). Coronaviruses as Vectors: Position Dependence of Foreign Gene Expression. Journal of Virology, 77(21), 11312-11323. doi:10.1128/jvi.77.21.1131211323.2003.

[11] Murray J.D. Spatial Models and Biomedical Applications (2003). Springer, Berlin - New York. ISBN 0-387-95228-4.

[12] Dimaschko, J., \& Podolsky, V. (2020). Viral Mutations as a Possible Mechanism of Hidden Immunization and Containment of a Pandemia. doi:10.1101/2020.04.09.20059782.

[13] Shaman, J., \& Galanti, M. (2020). Will SARS-CoV-2 become endemic? Science, 370(6516), 527-529. doi:10.1126/science.abe5960.

[14] Linde, A., Rotzén-Östlund, M., Zweygberg-Wirgart, B., Rubinova, S., \& Brytting, M. (2009). Does viral interference affect spread of influenza? Eurosurveillance, 14(40). doi:10.2807/ese.14.40.19354-en. 